\title{
An Alternative Method for Characterizing Capacitor Matching
}

\author{
KEE-CHEE TIEW, RANDALL GEIGER
}

E Cp E Department, Iowa State University, Ames, IA

Abstract - An alternative method for characterizing capacitor matching is presented. The basic idea of this method is to sense the mismatch among the capacitors by amplifying the error voltage using an iterative switched-capacitor scheme. Through simulation, this method has shown attractive property for sensing capacitor mismatches down to $1 \%$ and smaller.

\section{INTRODUCTION}

Switched-capacitor is one of the most popular approaches in data converters design. However, high-resolution data converters require accurate capacitor matching. In order to improve circuit performance, both digital and analog calibration techniques were proposed and discussed in the literature [1-5]. Among the many calibration methods, the under-range/ over-range method had been greatly discussed in the literature [1,5]. This method utilizes a capacitor array to correct mismatches by monitoring the under-range or over-range occurrence of the signal. However, it is difficult to detect under-range or over-range of the signal if the mismatch is considerably small. Therefore, it is the interest of this paper to discuss a method of picking up small signal that corresponds to small mismatch. By doing so, it is possible to achieve highly accurate capacitor matching. Figure 1 depicts a gain-of-two circuit that is widely used in pipelined and cyclic A/D converters. Note that the circuit only requires a ratio of one capacitor matching.

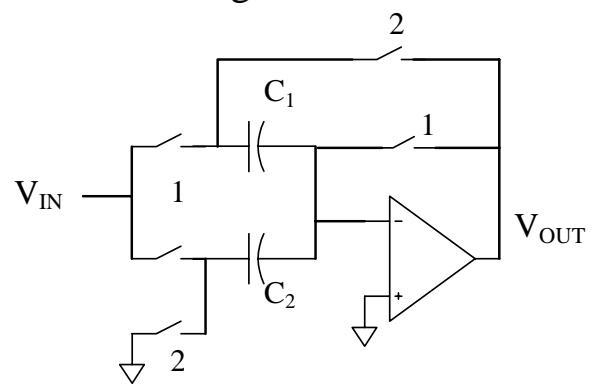

Figure 1: Gain-of-two circuit

The output, $\mathrm{V}_{\text {OUT }}$, of the circuit is given by the following expression:

$$
V_{\text {OUT }}=V_{I N}\left(1+\frac{C_{2}}{C_{1}}\right)
$$

If $\mathrm{C}_{2}=(1+\varepsilon) \mathrm{C}_{1}$ where $\varepsilon$ is 0 nominally, then (1) can be rewritten as

$$
V_{\text {OUT }}=V_{I N}(2+\varepsilon)
$$

From (2), one can see that the error due to capacitor mismatch has an expression of $\varepsilon \mathrm{V}_{\mathrm{IN}}$. If $\varepsilon$ is small, the calibrating circuit required to pick up the small mismatch error needs to be highly sensitive. To ease the requirement on the calibrating circuit, this paper is proposing a simple switched-capacitor scheme that is able to pick up small mismatch error. 
BASIC IDEA

The same switched-capacitor circuit in Figure 1 is reconfigured as depicted in Figure 2(a). This is the reset phase of the calibration, during which $\mathrm{C}_{1}$ is charged to $\mathrm{V}_{\mathrm{IN}}$ while $\mathrm{C}_{2}$ is discharged. In the next phase (phase 1) as in Figure 2(b), $\mathrm{C}_{1}$ is hold and $\mathrm{C}_{2}$ is charged to $\mathrm{V}_{\mathrm{IN}}$. If $\mathrm{C}_{1}$ and $\mathrm{C}_{2}$ are perfectly matched and everything else is ideal, then the charge transfer from $\mathrm{C}_{2}$ should completely cancel the charge held in $\mathrm{C}_{1}$. This will result in a zero voltage change at $\mathrm{V}_{\text {OUT }}$. Nevertheless, if there is a capacitor mismatch of $\theta$ between $C_{1}$ and $C_{2}$ $\left(C_{2}=\theta C_{1}\right.$ where $\theta$ is 1 nominally), a non -zero voltage change, $\Delta \mathrm{V}_{\text {OUT }}$, given by the following expression will result at $\mathrm{V}_{\text {OUT }}$.

$$
\Delta V_{\text {OUT }}=(1-\theta) V_{I N}
$$

The error voltage or $\Delta \mathrm{V}_{\text {OUT }}$ obtained at the output of $A_{1}$ is sampled by $C_{3}$ and the voltage is then held by $A_{2}$ in the next phase (phase 2) to charge up $\mathrm{C}_{2}$, as depicted in Figure 2(c). With the error voltage $(1-\theta) \mathrm{V}_{\text {IN }}$ stored in $\mathrm{C}_{2}$, the switched-capacitor will go through phase 1 again as in Figure 2(b), and followed by phase 2 as in Figure 2(c) for $k$-cycle. At the end of the $k^{\text {th }}$ cycle, the error voltage $\Delta \mathrm{V}_{\text {OUT }}$ has the following expression:

$$
\left.\Delta V_{\text {OUT }}\right|_{k-t h}=\left(1-\theta^{k}\right) V_{I N}
$$

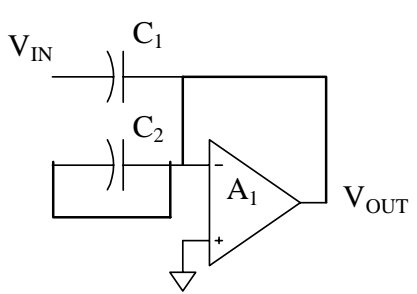

(a)

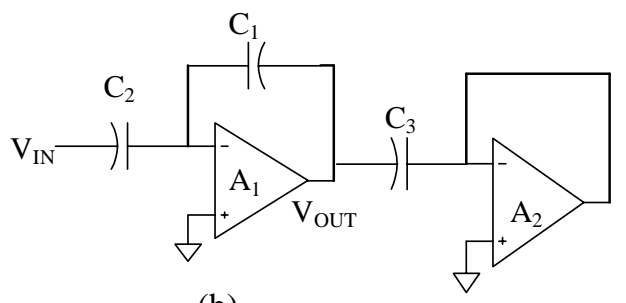

(b)

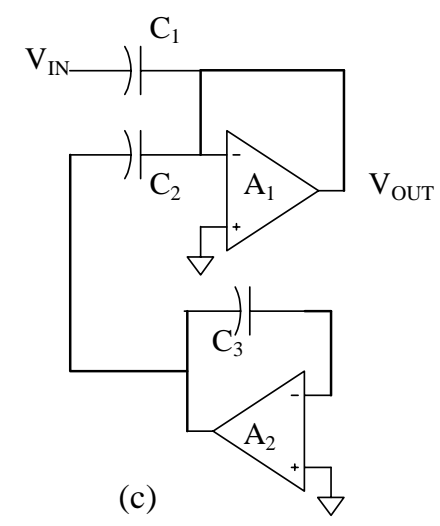

Figure 2 (a) Reset phase. $C_{1}$ is charged to $V_{I N}$; $\mathrm{C}_{2}$ is discharged. (b) Phase 1. Any mismatch between $\mathrm{C}_{1}$ and $\mathrm{C}_{2}$ will result in an error voltage $\Delta \mathrm{V}_{\text {OUT }} ; \mathrm{C}_{3}$ is charged to $\Delta \mathrm{V}_{\text {OUT. }}$ (c) Phase 2. This phase is similar to the reset phase except $C_{2}$ is charged to $\Delta \mathrm{V}_{\text {OUT }}$ instead of being discharged.

In Table 1 , one can see that $\Delta \mathrm{V}_{\text {OUT }}$ due to a mismatch of $\pm 0.5 \% \quad(\theta=0.995$ or $1.005)$ is amplified by approximately 10 at the $10^{\text {th }}$ iteration $(\mathrm{k}=10)$. The error voltage $\Delta \mathrm{V}_{\text {OUT }}$ due to $0.5 \%$ mismatch is $5 \mathrm{mV}$ in contrast to $50 \mathrm{mV}$ at the end of the $10^{\text {th }}$ cycle; hence it is almost trivial to build the comparator needed to pick up the much bigger error signal despite of the small mismatch. 


\begin{tabular}{|c|c|c|c|c|}
\hline$k^{\theta}$ & 0.995 & 0.99 & 1.005 & 1.01 \\
\hline 1 & 0.0050 & 0.0100 & -0.0050 & -0.0100 \\
\hline 2 & 0.0100 & 0.0199 & -0.0100 & -0.0201 \\
\hline 3 & 0.0149 & 0.0297 & -0.0151 & -0.0303 \\
\hline 4 & 0.0199 & 0.0394 & -0.0202 & -0.0406 \\
\hline 5 & 0.0248 & 0.0490 & -0.0253 & -0.0510 \\
\hline 6 & 0.0296 & 0.0585 & -0.0304 & -0.0615 \\
\hline 7 & 0.0345 & 0.0679 & -0.0355 & -0.0721 \\
\hline 8 & 0.0393 & 0.0773 & -0.0407 & -0.0829 \\
\hline 9 & 0.0441 & 0.0865 & -0.0459 & -0.0937 \\
\hline 10 & 0.0489 & 0.0956 & -0.0511 & -0.1046 \\
\hline
\end{tabular}

Table 1: Calculated $\Delta \mathrm{V}_{\text {OUT }}$ due to $\pm 0.5 \%$ and $\pm 1 \%$ mismatches for 10 cycles.

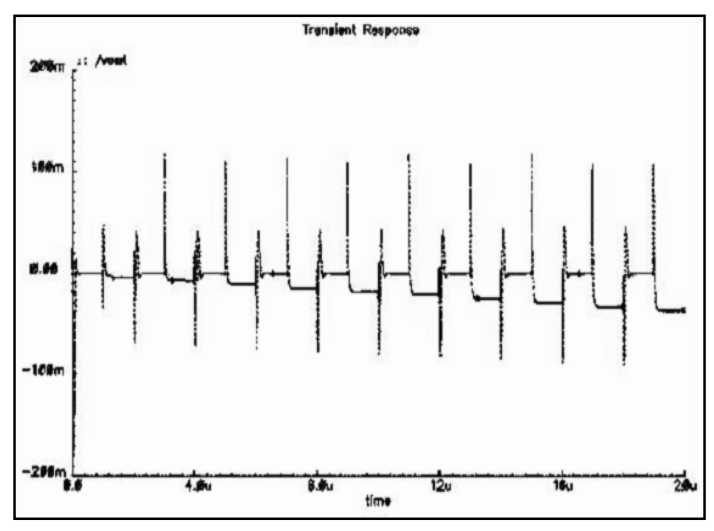

Figure 3: $\mathrm{V}_{\text {OUT }}$ transient waveform for $+0.5 \%$ mismatch.

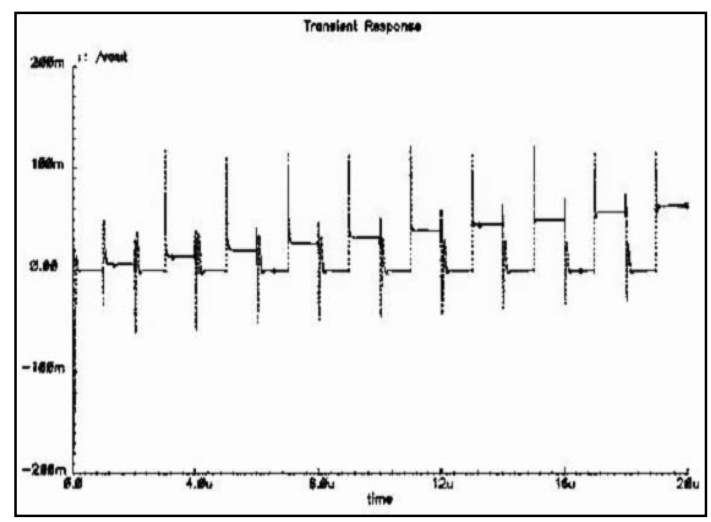

Figure 4: $\mathrm{V}_{\text {OUT }}$ transient waveform for $-0.5 \%$ mismatch.
Transistors level simulation using HPSICE was performed to verify the discussion. The circuit implementation is depicted in Figure 5. Minimum sized transmission gates were used to realize the switches while the op-amp has a DC gain of $82 \mathrm{~dB}$ and GB of 10MHz. Figure 3 and 4 depict the $V_{\text {OUT }}$ transient waveforms for 10 cycles with $\theta=0.995$ and 1.005. Note that $\Delta \mathrm{V}_{\text {OUT }}$ is amplified in every clock cycle. The simulated data for $\Delta \mathrm{V}_{\text {OUT }}$ can be found in Table 2. It can be seen that the simulated result is in close agreement with the calculated result. The error introduced by the op-amp and the switches sets an upper limit on the attainable accuracy of this calibration scheme.

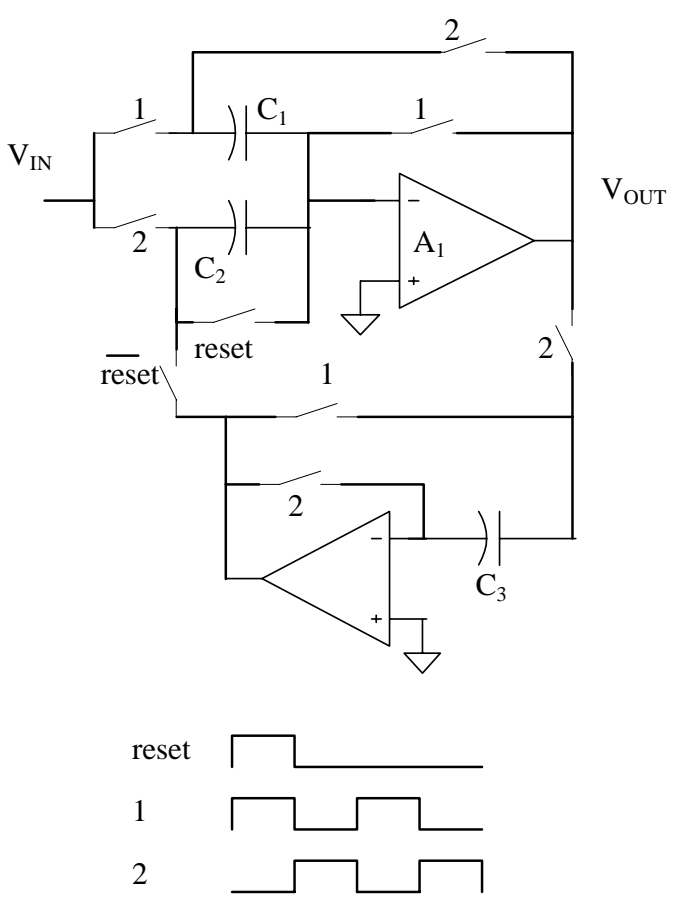

Figure 5: Circuit implementation and timing diagram. 


\begin{tabular}{|c|c|c|c|c|}
\hline $\mathrm{k}^{\theta}$ & 0.995 & 0.99 & 1.005 & 1.01 \\
\hline 1 & 0.0061 & 0.0112 & -0.0040 & -0.0088 \\
\hline 2 & 0.0127 & 0.0225 & -0.0070 & -0.0162 \\
\hline 3 & 0.0193 & 0.0341 & -0.0099 & -0.0243 \\
\hline 4 & 0.0258 & 0.0452 & -0.0135 & -0.0340 \\
\hline 5 & 0.0330 & 0.0559 & -0.0164 & -0.0416 \\
\hline 6 & 0.0397 & 0.0673 & -0.0191 & -0.0506 \\
\hline 7 & 0.0461 & 0.0783 & -0.0221 & -0.0591 \\
\hline 8 & 0.0532 & 0.0895 & -0.0263 & -0.0678 \\
\hline 9 & 0.0593 & 0.1007 & -0.0300 & -0.0773 \\
\hline 10 & 0.0662 & 0.1114 & -0.0330 & -0.0857 \\
\hline
\end{tabular}

Table 1: Simulated $\Delta \mathrm{V}_{\text {OUT }}$ due to $\pm 0.5 \%$ and $\pm 1 \%$ mismatches for 10 cycles.

\section{CONCLUSION}

A switched-capacitor scheme that can be used to characterize capacitor matching has been discussed. The major feature of this scheme is its ability to amplify the small error signal due to small capacitor mismatch. This scheme can be adopted in on-chip calibration and built-in self-test systems [6-8] while relieving the performance requirement of other circuits in the system.

\section{REFERENCES}

[1] H. Ohara, H. X. Ngo, M. J. Armstrong, C. F. Rahim, and P. R. Gray, “ A CMOS programmable self-calibrating 13-bit eight-channel data acquisition peripheral," IEEE $J$. Solid-State Circuits, vol. SC-22, pp. 930-938, Dec. 1987.

[2] H. T. Yung, K. S. Chao," An error-compensation A/D conversion technique,” IEEE Trans. Circuits Syst, vol. 38, No. 2, pp. 187-195, Feb. 1991.

[3] A. M. A. Ali, K. Nagaraj," Correction of operational amplifier gain error in pipelined A/D converters," ISCAS 2001 IEEE Int. Symp. Circuits Syst., vol. 1, pp. 568 -571, 2001.

[4] T. L. Sculley, M. A. Brooke," Nonlinearity correction techniques for high speed, high resolution A/D conversion," IEEE Trans. Circuits Syst. II: Analog and Digital Signal Processing, vol. 42-3, pp. 154 -163, Mar. 1995.

[5] K. Nagaraj," Efficient circuit configurations for algorithmic analog to digital converters," IEEE Trans. Circuits Syst. II: Analog and Digital Signal Processing, vol. 40-12, pp. 777 -785, Dec. 1993.

[6] E. J. Peralias, G. Huertas, A. Rueda, J. L. Huertas," Self-testable pipelined ADC with low hardware overhead," 19th. VTS 2001 IEEE Proc. VLSI Test Symp., pp. $272-277$, 2001.

[7] E. K. F. Lee," Reconfigurable data converter as a building block for mixed-signal test," 1997 Proc. European Design and Test Conference, pp. 359 -363, 1997.

[8] E. Teraoka, T. Kengaku, I. Yasui, K. Ishikawa, T. Matsuo, H. Wakada, N. Sakashita, Y. Shimazu, T. Tokuda,” A built-in self-test for $\mathrm{ADC}$ and $\mathrm{DAC}$ in a single-chip speech CODEC," 1993 Int. Proc. Test Conference, pp. 791 -796, 1993. 